THURSDAY, OCTOBER 29, 1885

\section{THE ANTI-CHOLERA INOCULATIONS OF DR. FERRAN}

$\mathrm{I}$ the spring and summer of the present year the public in Europe-lay and medical-have been greatly agitated by the exploits of a Spanish medical gentleman, who, during the cholera epidemic then raging in Spain, claimed to have discovered a means of preventing cholera. He was hailed as a great benefactor, and if his deeds had been equal to his professions, he would no doubt fully deserve to rank with Jenner, the greatest benefactor to mankind. But fortunately the medical world, at any rate the scientific medical world outside Spain, is not guided by the allegations of enthusiasts nor by wonder-doctors either. A Don Quixote, who discerns in a windmill giants, in a flock of sheep a squadron of the enemy's soldiers, may present points of interest to the psychologist: to the disciple of physiology and pathology he demonstrates an aberration of the visual nerve centres. I shall show that Dr. Ferran comes very near in rank, not to Jenner, but to his own illustrious countryman, the Knight of La Mancha.

The method of Ferran is practically this :-Ferran says that by a peculiar mysterious method of cultivationwhich for a long while he was not going to divulge--he has succeeded in attenuating the action of the comma bacillus of Koch. In these cultivations the comma bacillus after very complex morphological changes, unnecessary to detail here, forms spores. Such cultures introduced in sufficient quantities into the subcutaneous tissue of animals (guinea-pigs) or man produce a disease which is a mild and abortive form of cholera ; it manifests itself in local inflammation, and a general constitutional disturbance, febrile rise of the body temperature, headache, nausea, and sickness, and even diarrhœa. After a few days the person inoculated returns to his normal state. Persons once, twice, or thrice inoculated answer, or ought to answer, each inoculation with the said constitutional disturbance. Statistics collected by Ferran and his adherents in the places where these inoculations were practised, notably in Alcira, in and about Valencia, prove, so it is said, that the number of cholera cases and of deaths from cholera decreased in a conspicuous degree after these inoculations had been commenced, and also that those persons that had been inoculated remained almost impervious to cholera, while others not so inoculated fell victims to the plague in large numbers. In these assertions and practices several important questions are involved, each of which demands a direct answer, which ought to be favourable to this theory of Dr. Ferran.

First: Is the so-called cholera-bacillus, or Koch's comma-bacillus, found in the intestinal discharges of cholera patients, the vera causa of cholera?

Second: Does this so-called cholera-bacillus form spores, which when introduced into the living tissue germinate into the comma bacilli: in the subcutaneous tissue capable of producing only an abortive and mild form, but in the alimentary canal producing severe and malignant cholera?

VOL. XxxI1.-No. 835
Third : Do the cultivations of Dr. Ferran, when inoculated into the subcutaneous tissue, set up a disturbance which can be considered as an abortive form of cholera?

Fourth: Are persons so inoculated really protected or almost protected against an attack of real cholera; and do the statistics collected by Ferran and his adherents prove this?

(I) The first of these questions, it is obvious, forms the basis of the whole theory; for if the comma bacillus of Koch is not the real cause of cholera all the rest of Ferran's assertions, as far as cholera is concerned, fall to the ground. The claims of the comma bacillus of Koch to be accepted as the true cause of cholera, rests on very insufficient evidence: the epidemiological evidence as to the spread of cholera being dependent on soil and season, the anatomical evidence as to the comma bacilli being limited to the cavity of the cholera intestine, they being absent from the tissues and the blood, the misproportion existing between the number of comma bacilli present in the alimentary cavity, and between the severity and acuteness of the disease in many cases, and a number of other facts not necessary to mention here, prove to my mind that the comma bacillus is not the real cause of cholera. Add to this that Emerich of Munich vindicates this claim to be the real cause of cholera, not to the comma bacilli of Koch, but to small straight bacilli, probably identical with those seen and described by the English cholera Commission in India as constantly present in the alimentary canal of cholera patients, and for which bacilli I did not and cannot claim any real infective power; and further, that Emerich's view is backed up by no less an authority than Von Pettenkoffer himself, There is then at present an interesting contest going on between two rival bacilli: one, having Berlin for the head-quarters of its advocates, may be called the northern bacillus; the other, in Munich, may be called the southern bacillus. As to the actual facts, it seems to me the question is not whose claim is stronger, but whose claim is weaker.

(2) All except Ferran, acquainted practically with the comma bacillus in pure cultivations (Koch, Van Ermengem, myself, Mr. Watson Cheyne, Finkler, Emerich, Buchner, Klebs, and many others) are agreed that the comma bacillus in artificial cultivations never forms spores; having multiplied until all the nutritive material in the cultivation is exhausted, a period arrives when the comma bacilli degenerate and die; some undergo this long before the point of exhaustion is reached, others retain their vitality longer, but after weeks and months death has involved all the comma bacilli present in the cultivation. [An impurity accidentally present in the culture would effect this death of the comma bacilli in a much shorter period; in fact, in many instances, they would not have much chance of primarily reaching any considerable number.]

When this period has been reached, the culture becomes incapable of starting a new culture; and vic versâ : by this means the point of death of the bacilli present in the culture can be tested and accurately determined. I have a large number of tubes of pure cultivations of the comma bacilli, the nutritive medium being broth, or peptone and broth, or gelatine peptone and broth, or gelatine peptone and D D 
meat extract, or Agar-Agar peptone and broth, \&c. In each of these media the comma bacilli thrive well and form copious growths. The cultures are pure, contain the comma bacilli only, as all sub-cultures from them yield again the comma bacilli, and comma bacilli only. Now the remarkable fact about such culture tubes is this: that after several months all life in them becomes extinct, as is proved by inoculating from them a series of tubes containing suitable nutritive material, no comma bacillus or any other bacteria making their appearance. I have ascertained this in a great many cases, and it is in perfect agreement with the experience of Koch and many other workers. This clearly proves that there are not present in such tubes spores of the comma bacilli, for, if the comma bacilli, like some other bacilli-e.g. bacillus subtilis of hay infusion, or bacillus anthracis, were capable of forming spores, such a total extinction of life could not take place; the spores, although, owing to exhaustion of nutritive material, incapable of germinating into bacilli while in the tube in which they were formed, would undoubtedly germinate when transferred into a fresh and suitable nutritive medium. This total extinction of life does occur not only in tubes in which the nutritive medium is in a fluid condition, but also in all Agar-Agar peptone broth tubes, this material, unlike gelatine, remaining in its solid state, however luxuriant the growth of the comma bacilli may be.

Dr. Ferran claims to have discovered means by which the comma bacilli can be made to produce spores. In his cultures he notices a number of peculiar things which he considers as antecedents to the formation of spores and as fully formed spores. But direct observations that these are really spores, that, like spores, they actually germinate into the bacilli, Dr. Ferran has not deemed it necessary to make. As a matter of fact those to whom Dr. Ferran has shown his specimens, in which these alleged spores were supposed to be present, failed to see them (see the Report of the French Commission headed by Dr. Brouardel; see also Dr. van Ermengem's Report).

The methods of examination and cultivation of bacteria perfected by Koch, which, owing to the thoroughly reliable results they yield, are now universally followed by all who wish to acquire correct ideas and a sound knowledge of the life-history, morphology, and activity of bacteria, have led those practically acquainted with the comma bacilli to the conclusion that they do not form spores. Dr. Ferran is of the contrary opinion; but, judging from the Report of the French Commission, and from that.of van Ermengem and others, who have visited Ferran and seen him at work, it is pretty clear that this gentleman is not only unpractised in, but altogether unacquainted with the elements of technique necessary in bacterial investigations; more than this : according to a graphic description by the special correspondent of the Times, Dr. Ferran makes his cultivations in broth in a temporary laboratory, the kitchen of an untenanted house, reeking with the effluvia of an untrapped sewer opening into this kitchen. Dr. Ferran's cultivations have been examined microscopically by a Valencia Commission, who found that they contained a motley crowd of various kinds of bacteria; Dr. Chantemesse in a paper read before the Paris Académie de Médecine (see Brit. Med.
Fournal, Sept. 26, 1885) states that as the result of a microscopic examination of Dr. Ferran's cultures he found the fluid variable in its composition; sometimes it is a cultivation of impure comma bacilli, sometimes it contains masses of different micro-organisms, but the comma bacilli are barely present. Add to this that Dr. Ferran, as the special French. Commission attested, possesses neither the skill nor uses the ordinary precautions and apparatus indispensable in investigations of this nature, and all Ferran's extravagant assertions as to the behaviour of the comma bacillus in cultivations, as to its peculiar power of forming spores, must be regarded as sheer nonsense.

3. Notwithstanding this deficiency of Ferran in his mode of preparing his so-called "vaccine," it might be said, and it has been said by Dr. Cameron in a powerful and very able article in the Nineteenth Century for August 1885 , that by subcutaneously inoculating a cultivation of comma bacilli, no matter however impure and contaminated, e.g. such as were at Ferran's disposal, the effect is different from the one produced by introducing them into the alimentary canal. In the former case, i.e. in the subcutaneous tissue, they are planted in a soil not congenial to them, and their product is only an abortive form of cholera, whereas in the latter, i.e. in the cavity of the alimentary canal, they find a more suitable soil, a soil which is their natural breeding ground, and the result is virulent real cholera.

What Ferran by the inoculation of his cultures into the subcutaneous tissue of human beings actually did produce, is, according to a number of witnesses (see the letters of the special correspondent of the British Medical Fournal; the evidence given in detail by the special correspondent of the Times, October 20, and a number of other independent witnesses, English and French), septic infection, the intensity of which, as might be expected, and as Ferran himself admits, depends on the quantity injected. This result, however, is not always produced, the injection being sometimes quite inert, notwithstanding the presence of the comma-bacilli in the "vaccine" fluid. In the very able letter by the special corresponden $i$ of the Times for October 20 we are informed that Dr. Ferran explained to this gentleman in detail that the culture fluid used for inoculation need not contain any comma bacilli at all, in order to produce the desired result; further, that the comma bacilli can be killed by boiling or otherwise, without impairing the efficacy of the fluid, and that therefore a chemical substance present in the culture fluid, and probably the product of the organisms, must be regarded as the active principle. While this latest assertion of Ferran clearly shows that he is profoundly ignorant of the theory and practice of protective inoculations, such as are employed by Chauveau, Pasteur, Koch, Gaffky, Arloing and Thomas, and many others in a variety of specific diseases (anthrax, some forms of septicæmia, fowl cholera, symptomatic charbon, \&c.), and while it is in flagrant opposition to his own assertions of an earlier date, it also proves that the results obtained by Ferran by the inoculations of his "cholera vaccine" into the subcutaneous tissue of human beings harmonise well with the assumption that what he produces is simply septic poisoning, i.e. changes such as have been proved to follow the injection of certain chemical substances known 
as ptomaïnes, and produced by the growth and activity of putrefactive bacteria in media containing proteids. Brieger ("Die Ptomaine," Hirschwald, Berlin, I885) has published a most important series of observations on the production, nature, and action of ptomaines, and has greatly enlarged our knowledge of this as yet obscure subject. The description of the symptoms observable on persons inoculated by Ferran (as given by a variety of independent witnesses and by Ferran himself), can leave little doubt that the result of these inoculations is septic poisoning, in severe cases dangerous phlegmon and ulceration, and even death. This is also the opinion of a number of medical men (Spanish, English, and French) who have had the opportunity of seeing and examining such persons, as will be seen from the Report by the Special Commissioner of the British Medical Fournal, the Report by the Special Commissioner of the Times, the Report by the Special French Commission, and the Report of the Commission sent by the Spanish Government. Such being the case, the inoculations practised by Ferran and his coadjutors can have no possible prophylactic effect against cholera, even granting, for the sake of argument, that one mild attack of cholera protects against a second severe one, a question which is still sub judice, since some competent authorities maintain that such immunity, although holding good in a number of infectious maladies, does not apply to cholera.

4. Now, are persons inoculated by Ferran furnished with immunity against an attack of cholera? The statistics published by Dr. Ferran and his adherents on the marvellous effects of inoculation in Alcira, Valencia and other places, accepted by Dr. Cameron in his article above referred to, show us a picture of brilliant successes, favourably comparing and even surpassing the statistics of the effect of vaccination against smallpox. Those statistics collected by Ferran being endorsed by several medical men and other notabilities of the town of Alcira and elsewhere, Dr. Cameron cannot bring himself to regard as not revealing the truth; he cannot imagine that all these worthy people should conspire to pervert the truth and to prevent the truth from becoming known.

The correspondent of the Times in his letter, published October 20, gives a long list of places where the statistics published by the Ferranists are signed and stamped by the Alcalde of the place, the local judge, the priest, the resident doctors, and the notary; all duly signed and stamped. This Englishman, however, probably knowing what value to attach to the competency and veracity of all those worthies, examined the statistics for himself, and the result of his inquiry may be briefly summarised by saying that Dr. Ferran and his partisans have simply "cooked" those statistics. They have done these things: when a person who had been inoculated by Ferran did nevertheless become affected with cholera, and died of it, death was put clown as caused not by cholera but by some other disease; false entries were made as to persons who, having been inoculated, nevertheless died of cholera, were not entered as having been inoculated; persons have been registered as having been "vaccinated" by Ferran, but on inquiry were found to have died of cholera several days previous to the alleged "vaccination."

Add to this the fact that in Alcira, for instance, the inoculations and their wonderful effects had not com- menced until the population had abandoned the impure water supply ; that in some places many of the inoculated persons belonging to the well-to-do classes (a fee being paid for the inoculation) were therefore less exposed to infection, and those statistics become a gross farce and a shameless imposture. And this is practically the opinion of the Special Commission sent by the Spanish Government; this Commission has reported altogether unfavourably on these inoculations, declaring them barren of all scientific value, dangerous inasmuch as persons inoculated and suffering in consequence from a form of septic poisoning become more susceptible to infection from cholera and other diseases, and further condemning them as of no value in giving immunity against cholera.

The fact that Dr. Ferran and his associates took payment for the inoculations-thousands of persons were inoculated and reinoculated in Valencia and elsewhere, for each inoculation a fee of from 5 to 12 francs being charged-gives to the whole business a very ugly look. The Times correspondent (Times, October 20) does not therefore fully express the real value of Dr. Ferran when he says that he (Dr. Ferran) "is the dupe of illusions, conceived in ignorance."

E. KLEIN

\section{LIFE OF SIR WILLIAM ROWAN HAMILTON}

\section{Life of Sir William Rowan Hamilton, Royal Astronomer} of Ireland. By Robert Perceval Graves, M.A., SubDean of the Chapel Royal. Vol. II. pp. 7I9. With Portrait. (Hodges, Figgis, and Co.)

$\mathrm{N}$ a former number of this journal it was our duty to notice the first volume of the life of the illustrious Irish mathematician. We have now to congratulate $\mathrm{Mr}$. Graves on the completion of the second instalment of that great work which has evidently been to him a labour of love. This volume, like its predecessor, bears abundant testimony to the conscientious manner in which the author has sought to delineate a picture of Hamilton, told as far as possible by the letters from Hamilton to his friends and by extracts from his journal. We are again surprised at the extraordinary copiousness of the materials which were available.

The incidents in the life of Hamilton apart from his literary and scientific activity are but few. The last volume conducted us to the year 1832 , when Hamilton was in his twenty-seventh year. We had there seen the troubled course of his two earlier love affairs, and at the outset of this volume we are introduced to the third with Miss Bayly, to whom he was married in 1833 . His domestic happiness was in the course of years clouded over by the ill-health of his wife, though to the end he remained an attached husband, as she was an attached wife; two sons and one daughter were the issue of this union.

The reader of this work can hardly fail to be struck with the number and the worth of the friends to whom Hamilton was endeared; he possessed to a remarkable degree the power of transforming a casual acquaintanceship into a true and lasting friendship. His intimacy with Wordsworth has been already referred to, and was carried on by occasional letters and visits until the death of the poet. Among his other literary friends we may mention Maria Edgeworth, who writes to him (p. 384):- 